\title{
A pneumograph transducer based on a small moving-coil loudspeaker
}

\author{
JOEL K. LEVY and R. J. HELMER \\ Trinity University, San Antonio, Texas 78284
}

An inexpensive pneumograph transducer is described.

A common type of pneumograph used in polygraph instrumentation and other human physiological monitoring makes use of a small rubber bellows in the form of an extensible cylinder which is coupled by flexible tubing to a piezoelectric transducer. A string around the chest of the $S$ is attached to each end of the bellows, which then expands and contracts with a volume change on the order of $1 \mathrm{cc}$. The piezoelectric device produces an electrical signal in response to the change in pressure which is due to the change in volume of the bellows. The pressure-to-voltage transducer may be a crystal microphone element mounted in an air-tight container coupled to the pneumatic system. Careful handling of this device is required because the conical foil diaphragm, whose vertex impinges on the surface of the crystal, is easily bent out of shape. This can happen under pressures generated by attaching the bellows to a $S$ unless one always remembers to open the system to outside air by means of a small valve provided for the purpose. It has been necessary for us to have such equipment operated by nontechnical personnel, and the short life of our first crystal unit suggested the study of magnetic types of transducers, which are potentially less subject to damage. We have been able to adapt a small inexpensive loudspeaker to this requirement with gratifying results. It is felt that the ruggedness and dependability of the unit are well worth the effort required to provide the special electronic circuitry required for signal conditioning.

The cone of a 2-in. 8-ohm loudspeaker (Radio Shack 40-245) was coated with a substance to seal the cone assembly airtight. A suitable coating was found to be acrylic polymer glossy medium used in acrylic painting (available through art supply houses as Liquitex; Permanent Pigments, Inc.). It remains stable and pliable and seals the porous material of the cone well enough for the requirement. Two circular pieces of $1 / 4$-in. Plexiglas were used to sandwich the speaker, and the seal at the rim of the cone was made airtight with a high-vacuum grease sealing compound containing silicones (Dow Corning Corp.). A polyethylene tube fitting was inserted in the hole of the Plexiglas facing to provide an attachment for the tubing leading to the bellows. A test was run to determine air leak over an arbitrary period of $1 \mathrm{~min}$. The results showed a $25 \%$ loss, which indicates an adequate sealing for the breathing rates of human Ss. The measurement was a test of the pneumatic system as a whole, and it was not considered worthwhile to determine what part of the leakage was through the speaker. The key to successful construction of the device lies in properly sealing the paper cone material without stiffening it.

If the $\mathrm{E}$ wishes to use the transducer with existing physiological monitoring equipment, we would encourage a certain amount of experimentation, depending on the flexibility of his equipment. If there is available a stable, high-gain amplifier, it may be that the addition of a simple $\mathrm{R}-\mathrm{C}$ circuit will provide the desired signal conditioning. Since the voltage output of the voice coil is proportional to the rate of change of volume of the pneumatic system, it is necessary to provide an integration at some point in the signal conditioning process. We have operated the transducer with a passive integrating circuit made up of a series resistor of $510 \mathrm{~K} \mathrm{ohms}$ and a capacitance of $50 \mathrm{mF}$ (Aerovox

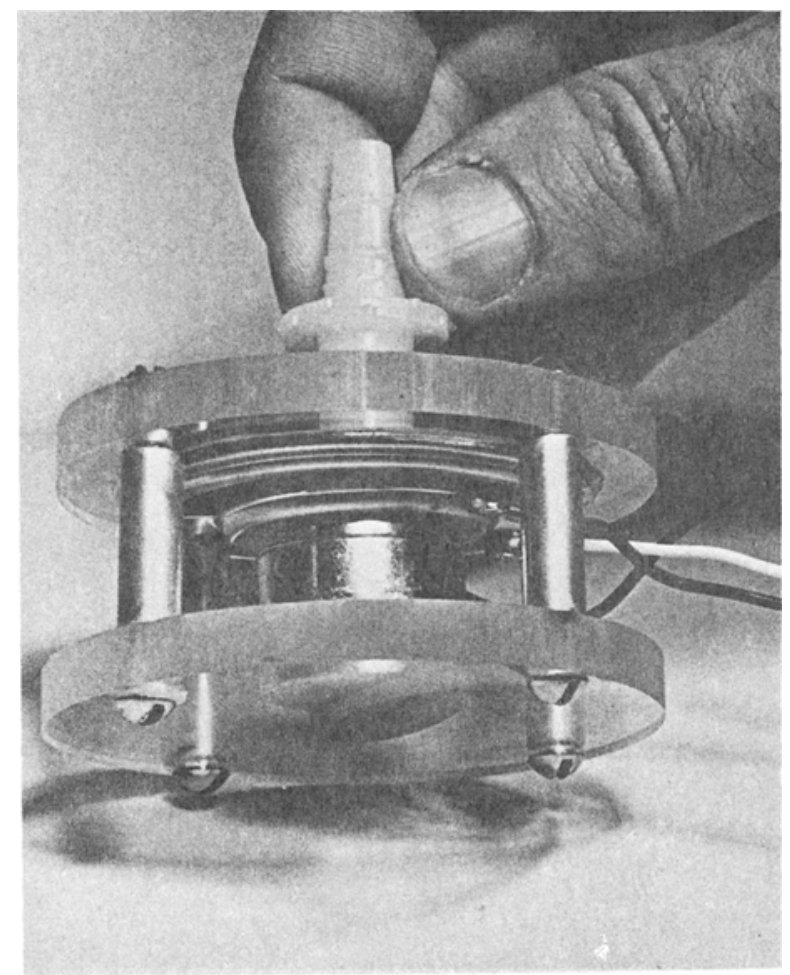

Fig. 1. Completed transducer assembly. Spacers are $3 / 4$ in. long. Note sponge rubber pad under speaker magnet. 


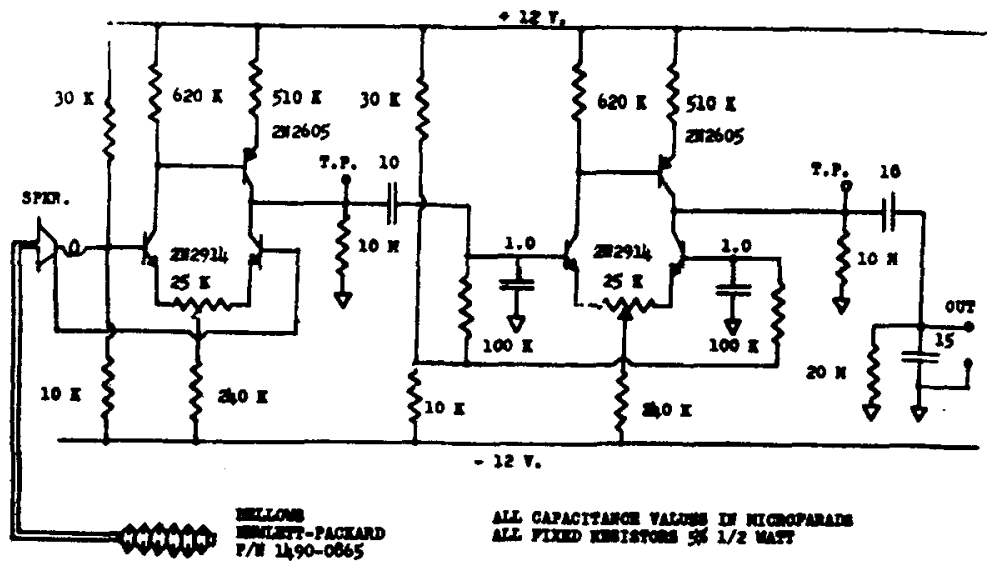

Fig. 2. A suitable preamplifier-integrator circuit for use with high input impedance postamplifier.

P09ZNR) across which the output is taken. The amplifier used to amplify the output of the integrator (Hewlett-Packard 8803) has an input impedance of 1 megohm or more, and sufficient gain to amplify the 10- to 20-microV input signal to the level required for display. If such an amplifier is not available, the preamplifier circuit of Fig. 2 is suggested as a means of interfacing with a wider variety of equipment. Here, preamplification is provided by two similar differential stages with ac coupling and very long time constants. This will allow use of the transducer without the need for a high-gain chopper-stabilized amplifier. The preamplifier output is taken across the integrating capacitor. The following amplifier should have an input impedance of $1 \mathrm{megohm}$ or more and must provide sufficient gain to raise a 5- to $10-\mathrm{mV}$ signal to the level required for display. The preamplifier should be constructed using mylar or other low-leakage-type capacitors. The values are not at all critical. The $10-\mathrm{mF}$ coupling capacitors and the $15-\mathrm{mF}$ integrating capacitor shown in the diagram were made up by connecting in parallel various capacitors of government surplus origin which happened to be at hand. The circuit will operate

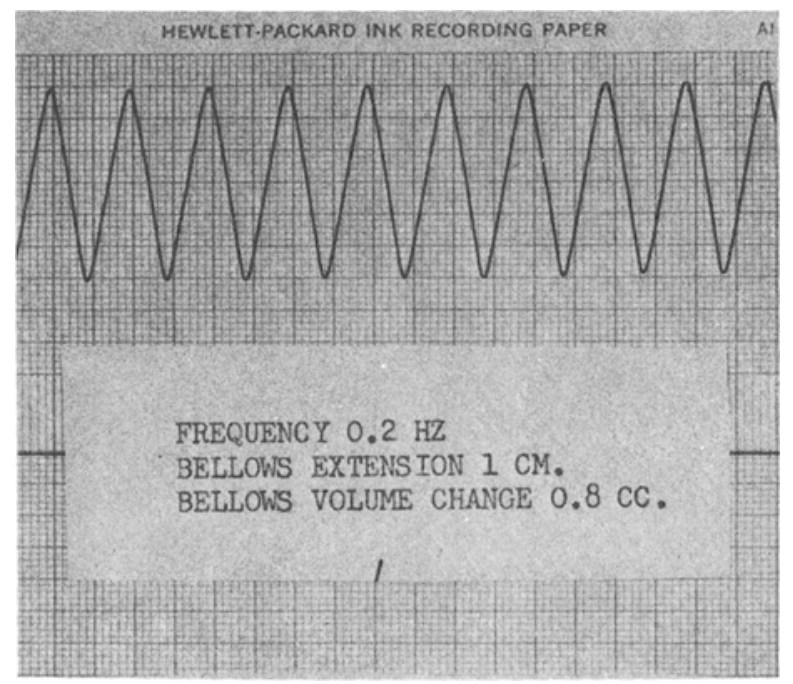

Fig. 3. Recording made with transducer and the circuit of Fig. 2, using isosceles sawtooth mechanical drive of bellows. satisfactorily with either batteries or simple Zener diode regulators as power sources. The preamplifier was built on an aluminum plate with one stage on each side and the assembly enclosed in an aluminum box. All components should be rigidly mounted rather close to the plate so that they will all tend to assume the same temperature. Because of the very long time constants, several minutes should be allowed for the circuit to reach its operating point after power has been applied. On initial operation, the balancing potentiometers should be adjusted to give $0 \mathrm{~V}, \pm 1 \mathrm{~V}$ or so, as measured with a high-impedance meter or oscilloscope probe at the test points designated TP.

A recording made with this equipment is shown in Fig. 3. For this, a low-frequency function generator supplying an isosceles triangle waveform was used to drive an $X-Y$ recorder to a $1-\mathrm{cm}$ peak-to-peak displacement along one axis only. One end of the bellows was attached by a thread to the pen carrier of the recorder so that a known mechanical signal was applied, with amplitude somewhat greater than the equipment is usually required to handle.

Those who wish to investigate the use of the transducer with other amplifiers should note that it is generally desirable, from the standpoint of stability, to use as large a capacitor in the integrating circuit as is economically feasible, with a correspondingly small resistance for a given time constant. Of course, the equivalent resistance value depends on the amplifier input impedance. Lastly, amplifier and integrator configurations other than those described here may be advantageous, depending on the type of equipment available.

\section{NOTE}

1. The transducer assembly of Fig. 1 can be built for about $\$ 4$. The replacement cost of the bellows used with it is \$15, but this item is not usually sold alone. We believe that other firms would provide a suitable substitute for about $\$ 5$, but we have not asked for quotations. The preamplifier of Fig. 2, without power supply, can be built for about $\mathbf{5 0}$ using a number of Aerovox V146Z-171 5-mF capacitors to make up the required values. If one wishes to make use of a supply of salvaged capacitors of the solid dielectric type, as we have done here, the above cost can be reduced by half.

(Received for publication July 18,1972 ; revision received October 11,1972 .) 